\title{
TENDENCIAS DE INVESTIGACIÓN EN PREECLAMPSIA ENTRE 1985-2013 EN EL PERÚ: UN ANÁLISIS BIBLIOMÉTRICO
}

\author{
Oscar Huapaya-Huertas ${ }^{1, a}$, Horacio Chacón-Torrico, ${ }^{1, a}$, Carolina Black-Tam²,b ${ }^{2}$ Hans Contreras-Pulache ${ }^{1, c}$, \\ Elizabeth Mori-Quispe ${ }^{1,3, d}$, Delia Indira Chiarello ${ }^{4, e}$
}

\begin{abstract}
RESUMEN
Objetivo. Caracterizar la producción científica nacional sobre preeclampsia durante el periodo 1985-2013. Materiales y Métodos. Análisis bibliométrico de los artículos de investigación realizados en Perú acerca de preeclampsia. Se realizó una búsqueda de revistas indizadas en PubMed, SciELO y LILACS desde 1985 hasta julio del 2013. Se seleccionaron investigaciones cuyo formato sea artículo original y que hayan sido realizadas en su totalidad en el Perú. Resultados. Se encontraron 32 artículos sobre preeclampsia, con un promedio de una publicación por año. El tiempo promedio entre la finalización del estudio y su publicación fue de $3,6 \pm 1,9$ años. El diseño de investigación más frecuente fue de caso-control $(53,1 \%)$, seguido de transversales $(21,8 \%)$, longitudinales $(15,6 \%)$ y por cohortes $(9,3 \%)$. Solo un $18 \%$ fue publicado en revistas científicas peruanas y el lugar más frecuente de elaboración del estudio fue Lima $(82,1 \%)$. Se encontró colaboración extranjera en el $50 \%$ de los artículos. Solo en 10 artículos se identificó el factor de impacto, encontrándose una media de 2,696. Conclusiones. La producción científica realizada en el Perú en torno a la preeclampsia resulta escasa en el periodo comprendido entre 1985 y 2013. Se precisa de modo urgente la implementación de estudios en este campo a fin de caracterizar el fenómeno en nuestro país. Esta implementación de estudios, se resalta, debe ser de carácter descentralizada y no focalizar solamente a la capital.
\end{abstract}

Palabras clave: Mortalidad materna; Eclampsia; Bibliografía estadística; Perú. (Fuente: DeCS BIREME)

\section{RESEARCH TRENDS IN PREECLAMPSIA BETWEEN 1985-2013 IN PERÚ: A BIBLIOMETRIC STUDY}

\begin{abstract}
Objective. The aim of the study is to characterize the national scientific production in preeclampsia during the period 19852013. Materials and Methods. Bibliometric analysis of scientific articles about preeclampsia in Peru. A search of journals indexed on PubMed, SciELO and LILACS from 1985 until July 2013. Original articles and those which have been done entirely in Peru were selected. Results. 32 articles on Preeclampsia were found, with an average of one publication per year in the studied period. The average time between the end of the study and its publication was $3.6 \pm 1.9$ years. The most frequent research design was case-control $(53,1 \%)$, followed by transversal $(21,8 \%)$, longitudinal $(15,6 \%)$ and by cohorts $(9,3 \%)$. Only $18 \%$ were published in Peruvian scientific journals and the most common location of study was Lima ( $82.1 \%)$. Foreign collaborations were found in $50 \%$ of the cases. The impact factor was identified in 10 articles with an average of 2.696. Conclusion. The scientific production in Peru around preeclampsia was scarce in the period between 1985 and 2013. There is an urgent need for the implementation of studies in this field in order to characterize the phenomenon in our country, in a decentralized mode and not focusing only in the capital.
\end{abstract}

Keywords: Maternal mortality; Eclampsia; Statistical bibliography, Peru. (Source: MeSH NLM)

\section{INTRODUCCIÓN}

La preeclampsia es un síndrome exclusivo del embarazo humano, y es definida por la presencia de hipertensión (niveles mayores de $90 \mathrm{~mm}$ de $\mathrm{Hg}$ o más de presión diastólica, y $140 \mathrm{~mm}$ de $\mathrm{Hg}$ o más de presión sistólica), proteinuria y, edema generalizado en embarazadas después de la semana 20 de gestación ${ }^{(1)}$. Sin embargo, aún con valores inferiores de presión arterial, se puede sospechar preeclampsia cuando se presentan incrementos iguales o mayores de $30 \mathrm{~mm}$ de $\mathrm{Hg}$ sistólicos y $15 \mathrm{~mm}$ de $\mathrm{Hg}$ diastólicos, con respecto a las cifras promedio registradas en la primera mitad del embarazo ${ }^{(2,3)}$. Concomitantemente con la hipertensión se presenta proteinuria y edema ${ }^{(4,5)}$. La proteinuria es definida como una excreción de proteínas en orina superior a los $300 \mathrm{mg}$ en 24 horas ${ }^{(6)}$. El edema representa la acumulación excesiva de líquido en el tejido subcutáneo, que conlleva a aumento de volumen de los tegumentos y a desaparición de los pliegues cutáneos. En general, el edema, en la mujer preeclámptica, es más severo y difuso que el que acompaña con frecuencia al embarazo normal. Este síndrome puede progresar y la paciente puede presentar insuficiencia renal, accidente cerebro vascular, daño hepático, edema pulmonar, coagulopatía y eclampsia (convulsiones)(7). Esta complicación se presenta, a nivel mundial, entre el $3 \%$ y $5 \%$ de todos los embarazos y es una de las causas principales de morbi-mortalidad materna(8). Este valor

\footnotetext{
Facultad de Medicina Humana. Universidad Científica del Sur . Lima- Perú.

Facultad de Psicología. Universidad Científica del Sur .Lima-Perú.

Facultad de Medicina Humana. Universidad Nacional Mayor de San Marcos . Lima-Perú.

Instituto Venezolano de Investigaciones Científicas.

Estudiante de Medicina Humana; ${ }^{b}$ Estudiante de Psicología; ${ }^{\circ}$ Médico Cirujano. Especialista en Salud Pública; ${ }^{d}$ Médico Cirujano. Especialista en Epidemiología e. PhD en Bioquímica.
}

Recibido: $10-10-13$

Aprobado: 3-12-13 
aumenta considerablemente en los países en vías de desarrollo como el Perú(9).

El análisis de la Encuesta Demográfica y de Salud Familiar (ENDES), del Instituto Nacional de Estadística e Informática (INEI), del año 2011 , estimó que el $73 \%$ de las muertes maternas estaban relacionadas a condiciones obstétricas, figurando entre estas la preeclampsia como la segunda causa principal (la primera está representada por las hemorragias postparto) ${ }^{(10)}$. Por tal motivo, en el marco del establecimiento de políticas en salud materna, el Ministerio de Salud del Perú (MINSA) estableció el "Plan Estratégico Nacional para la Reducción de la Mortalidad Materna y Perinatal 2009-2015", mediante el cual se propone reducir las posibilidades de complicaciones durante el embarazo, parto y puerperio, enfocándose así en la identificación de signos de alarma y de medidas de prevención de complicaciones en la gestante. Dichas medidas favorecerían la detección temprana de preeclampsia(11). En el año 2009, el MINSA, a través del Instituto Nacional de Salud (INS), promovió la identificación de las prioridades nacionales y regionales de investigación en salud para el período 2010-2014. Como resultado de este proceso participativo, se incluyó dentro de las prioridades nacionales de investigación el título de "Evaluaciones de impacto de estrategias e intervenciones actuales en mortalidad materna", por medio del cual se busca generar conocimiento que termine incidiendo en decisiones de salud pública, de tal forma que permitan disminuir las principales causas de mortalidad materna ${ }^{(12)}$.

Por otro lado, entre los Objetivos de Desarrollo del Milenio establecidos por la Organización de las Naciones Unidas (ONU), se encuentra que en relación a la salud materna, se debe disminuir la razón de muerte materna al 66,3 defunciones por cada cien mil nacidos ${ }^{(13)}$, es decir, reducir esta tasa en un $75 \%$ para el $2015^{(10)}$. Todas estas metas trazadas a nivel tanto internacional como nacional, que priorizan la investigación materno-infantil, indican la pertinencia de realizar investigaciones más exhaustivas e intervenciones en los temas de mayor relevancia, como es la preeclampsia.

Se entiende por análisis bibliométrico a aquel estudio en el cual de forma cualitativa y cuantitativa se evalúa la visibilidad, relevancia académica, evolución e impacto de los aportes científicos en un tema en específico ${ }^{(14)}$. Si bien existen estudios bibliométricos en Perú (15-17), no se encontró alguno que evalúe la investigación en alguna rama de la salud materno-infantil en general o de la preeclampsia en particular. Cabe mencionar que tampoco se han encontrado estudios bibliométricos a nivel latinoamericano o mundial que caractericen la literatura científica en preeclampsia, hecho por demás sorprendente conociendo la importancia de este trastorno y la cantidad de investigación que se implementa en este campo.

Con el propósito de analizar la evolución de la producción científica en un período de 28 años (1985-2013), se realizó un estudio bibliométrico que se espera contribuya a, primero, conocer el estado del arte de la investigación en preeclampsia en nuestro país y, segundo, servir de guía para próximas investigaciones en este campo.

\section{METODOLOGÍA}

Para el presente estudio bibliométrico se consideró a todos los artículos de investigación desarrollados en el Perú sobre el tema preeclampsia/eclampsia. Se realizó una búsqueda bibliográfica entre junio y julio del 2013 en las bases de datos PubMed, SciELO y LILACS. Se determinó como criterio de exploración aquellas bases académicas cuya cobertura y rigurosidad científica se rijan por la calidad de indexación de las revistas. Además, se consideró solo a aquellos artículos realizados íntegramente en el Perú, al margen del tipo de artículo (descriptivo o analítico), incluyendo estudios multicéntricos, independientemente de la nacionalidad de los investigadores. Se consideraron términos de búsqueda en inglés y castellano generados por términos clave como: "Preeclampsia and Perú", "Preeclampsia/eclampsia and Perú", "Preeclamptic women Perú", "Preeclampsia y Perú", "Preeclampsia/eclampsia y Perú". Estos términos debían estar incluidos en el título de la investigación y/o en las palabras claves. Se hizo además una búsqueda personalizada con los autores de investigaciones realizadas en el Perú, en busca de otras publicaciones afines a los objetivos de esta investigación. Se excluyeron artículos de revisión, cartas al editor, informes técnicos, tesis, libros y resúmenes de congresos y simposios. Los autores de esta investigación y expertos en el tema de preeclampsia realizaron la selección final de los artículos, a modo de respaldar el control de calidad.

La base de datos se elaboró utilizando el programa MS Excel 2013 para Windows, y se realizó el registro de la información según las siguientes variables: nombres de los autores, año de realización del estudio, año de publicación, tiempo de demora de aceptación en meses, tiempo desde la elaboración hasta la publicación en años, nombre de lar evista, tipo de estudio, idioma, lugar donde se realizó el estudio, instituciones participantes y factor de impacto de la revista científica. Posteriormente, se realizó un análisis descriptivo (frecuencias y porcentajes) con las variables categóricas y se emplearon medidas dispersión y tendencia central en las variables continuas; éste análisis se realizó con el programa SPSS 21. Para realizar el análisis de redes de investigación se utilizó el software de manipulación y visualización de gráficos Gephi 0.8.2.

\section{RESULTADOS}

Se encontraron 32 artículos sobre estudios en preeclampsia realizados en el Perú desde 1985 hasta julio del 2013. De la búsqueda en la base de datos PubMed se obtuvo 18 artículos, 5 en SciELO, y 14 en LILACS, según los criterios descritos previamente. Hubo 2 artículos que estuvieron adscritos tanto en PubMed como en 


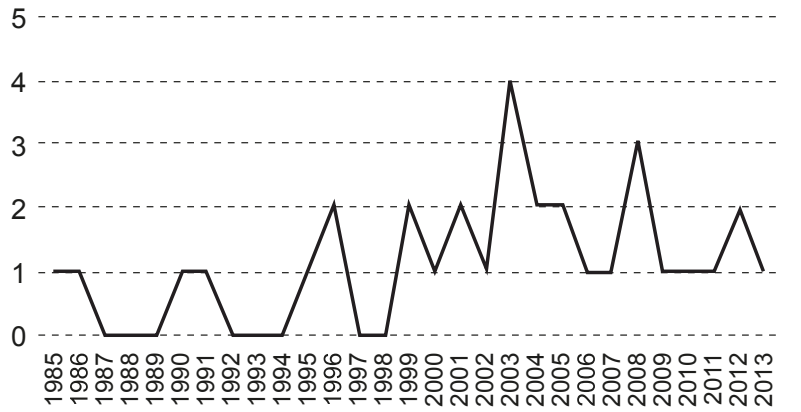

Figura 1. Número de artículos científicos sobre preeclampsia en el Perú, período 1985-2010.

SciELO, y 3 que se encontraron en PubMed y LILACS simultáneamente. Durante el lapso de tiempo estudiado, se registró que el máximo número de publicaciones por año fueron 4, cifra alcanzada en el 2003; y un mínimo de cero publicaciones en un total de 11 años. En la figura 1 se pueden observar estas tendencias de publicación.

De los 32 artículos analizados, en 27 se pudo determinar el tiempo de demora entre la finalización del estudio hasta su publicación, hallándose una media de 3,6 $\pm 1,9$ años, dentro de un rango de 1 hasta 8 años como tiempo máximo (Figura 2).

Por otro lado, solo se pudo determinar en 16 artículos lo concerniente al tiempo de aceptación por parte de las revistas. Así, en promedio, una revista demora 4,5 $\pm 2,7$ meses; el mayor tiempo transcurrido entre el envío y aceptación fue de 12 meses ( 1 artículo) y el menor tiempo fue de un mes (2 artículos).

Del total de artículos, 18 fueron publicados en inglés. El diseño de investigación más frecuente fue de caso-control, que corresponde a 17 de los estudios encontrados, 7 de ellos fueron transversales, 5 longitudinales y la diferencia, entre cohortes y cohortes retrospectivas, representaron 3 investigaciones.

De las 32 publicaciones analizadas, sólo 9 fueron publicadas en una revista científica peruana. El lugar más frecuente donde se realizaron los estudios fue en Lima/ Callao, lugar en que se llevaron a cabo 26 estudios del

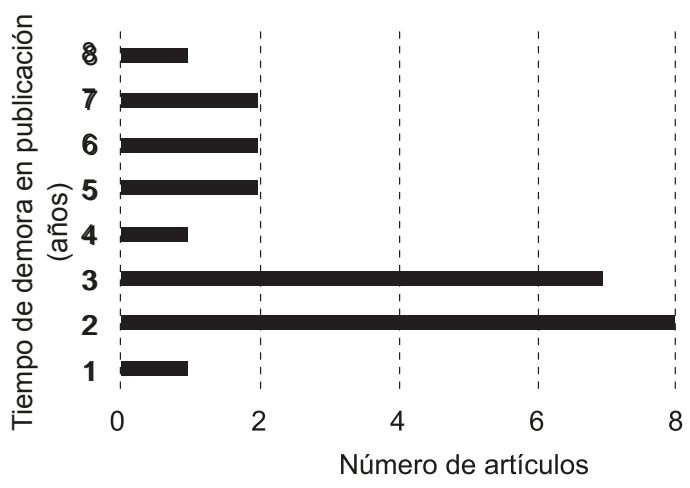

Figura 2. Tiempo demora (en años) en la publicación de artículos científicos sobre preeclampsia en el Perú, 1985-2010. total de las investigaciones. En las regiones Trujillo y Arequipa, se realizaron 2 investigaciones, en otras 2 no se especificó la región y se halló 2 estudios multicéntricos a nivel nacional.

Por otro lado, se registró que en 16 de las publicaciones seleccionadas hubo participación internacional y en sólo una no hubo colaboración nacional alguna. Las instituciones peruanas y extranjeras con mayor participación en la investigación sobre preeclampsia en el Perú, en el período estudiado, fueron el "Instituto Nacional Materno Perinatal", con 12 publicaciones; el "Center for Perinatal Studies, Swedish Medical Center" con 10 publicaciones; el "Hospital Nacional 2 de Mayo" con 8 publicaciones; y la "Universidad de Washington" con 8 publicaciones. En cuatro de los estudios no se pudo identificar a las instituciones participantes. La tabla 1 muestra las instituciones nacionales e internacionales con publicaciones sobre preeclampsia en el Perú y el factor de impacto de las revistas biomédicas en las que se publicaron las investigaciones.

Para comparar y evaluar la relevancia relativa de las publicaciones seleccionadas, según en número citaciones en el período de tiempo definido, se obtuvo el factor de impacto mediante el Journal Citation Reports, bajo los estándares del Instituto para la Información Científica (ISI- Institute for Scientific Information). Se pudo determinar el factor de impacto en sólo 10 de las revistas en las que fueron publicadas las investigaciones. E líndice promedio fue de 2.696 siendo el menor valor de 1.103 correspondiente a la revista "Gynecologic and Obstetric Investigation" y el mayor factor de impacto alcanzado fue

Tabla 1. Instituciones nacionales e internacionales que han publicado artículos científicos sobre preeclampsia en el Perú.

\begin{tabular}{|c|c|}
\hline Instituciones Nacionales & $\begin{array}{c}\mathrm{N}^{\circ} \mathrm{de} \\
\text { publicaciones }\end{array}$ \\
\hline Instituto Nacional Materno Perinatal & 12 \\
\hline Hospital Nacional Dos de Mayo & 8 \\
\hline Universidad Cayetano Heredia & 6 \\
\hline Universidad Nacional Mayor de San Marcos & 4 \\
\hline Hospital Guillermo Almenara Irigoyen & 1 \\
\hline Hospital Nacional Arzobispo Loayza & 1 \\
\hline Universidad Nacional San Agustín & 1 \\
\hline Hospital Nacional del Sur & 1 \\
\hline Universidad Norbert Wiener & 1 \\
\hline Centro de Investigación Anastomosis & 1 \\
\hline Instituciones extranjeras & $\begin{array}{c}\mathrm{N}^{\circ} \text { de } \\
\text { publicaciones }\end{array}$ \\
\hline $\begin{array}{l}\text { Center for Perinatal Studies, Swedish Medi- } \\
\text { cal Center }\end{array}$ & 10 \\
\hline Universidad de Washington & 8 \\
\hline $\begin{array}{l}\text { Division of Clinical Research, Fred } \\
\text { Hutchinson Cancer Research Center }\end{array}$ & 3 \\
\hline $\begin{array}{l}\text { Department of Obstetrics and Gynecology, } \\
\text { Loyola University Medical Center }\end{array}$ & 1 \\
\hline Oregon Regional Primate Research Center & 1 \\
\hline Universidad Nacional Autónoma de México & 1 \\
\hline
\end{tabular}


Tabla 2. Factor de Impacto de las revistas donde se publicaron los artículos seleccionados sobre preeclampsia

\begin{tabular}{lc}
\hline \multicolumn{1}{c}{ Revista } & $\begin{array}{c}\text { Factor de } \\
\text { impacto }\end{array}$ \\
\hline American Journal of Epidemiology & 4.780 \\
\hline American Journal of Hipertension & 3.665 \\
American Journal of Obstetrics and Gynecology & 3.117 \\
\hline Journal of Reproductive Immunology & 2.342 \\
BMC Womens Health & 1.505 \\
Archives of Gynecology and Obstetrics & 1.330 \\
Gynecological Endocrinology & 1.303 \\
\hline Gynecologic and Obstetric Investigation & 1.103 \\
\hline
\end{tabular}

de 4.78 del "American Journal of Epidemiology", como se observa en la tabla 2.

En cuanto a las redes de investigación en el tema preeclampsia, se encontró que el mayor porcentaje de estudios peruanos han sido realizados por grupos de investigación, entre los cuales se destaca la labor de S. Sánchez, siendo autor principal de 8 trabajos en el período de 28 años. Estas redes de trabajo científico se pueden observar en detalle en la figura 3 que representa la interconexión de profesionales abocados al trabajo científico en el tema. Los nodos corresponden al autor principal, y el tamaño del nombre del autor es proporcional al número de participaciones como tal. Los enlaces representan a los colaboradores secundarios del artículo, que a su vez tienen conexión con autores de otros estudios.

\section{DISCUSIÓN}

El presente artículo pretende mostrar un análisis descriptivo acerca de las publicaciones sobre preeclampsia realizados en el Perú en los últimos 28 años. Desde la publicación del primer artículo en 1985, se observa una alarmante carencia de producción literaria sobre esta temática.

En cuanto al número de publicaciones por año, si bien hay una razón aproximadamente un poco más de una por

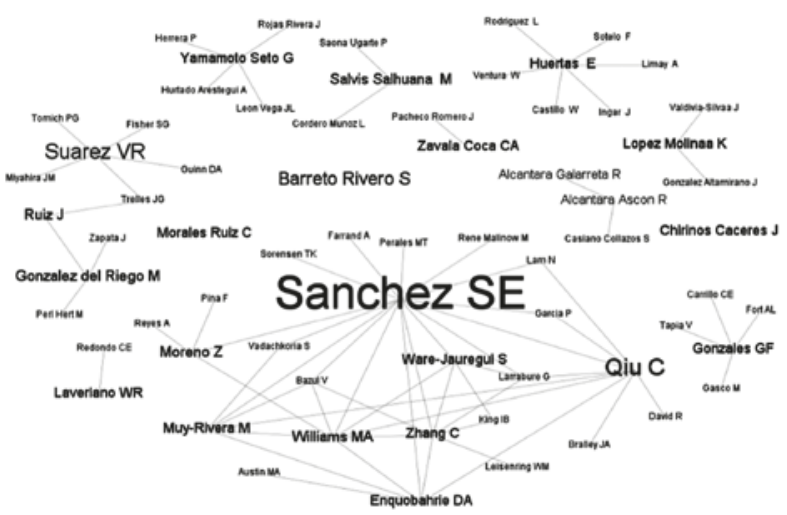

Figura 3. Redes de investigación sobre preeclampsia en el Perú año, se encuentra que las tendencias son heterogéneas, encontrándose picos, por ejemplo, de 4 publicaciones en el año 2003 y una nula producción entre los periodos: 1987-1989, 1992-1994 y 1997-1998. Cierto es que los dos primeros periodos mencionados representan en la historia de nuestro país dos de los periodos socialmente más comprometidos en términos de estabilidad social, política y económica (en el primero podemos ubicar la mayor crisis inflacionaria en toda la historia del Perú $y$ en el segundo consignaremos el ajuste estructural que cambió el modelo económico en nuestro país). En apariencia, éstas podrían ser las causas por las cual no se registran publicaciones en estos años. Sin embargo, al comparar nuestra investigación con otros análisis bibliométricos de similar relevancia académica realizados en el Perú, se encuentran uno sobre $\mathrm{VIH} /$ SIDA ${ }^{[12]} y$ otro sobre tuberculosis [11]. En el primero, se analizaron 257 publicaciones en un intervalo de 25 años; en el segundo, se encontró que fueron 233 en 29 años. Resultaría interesante, a partir de esta observación, la realización de un estudio que justamente determine el nivel de afectación en la producción científica en relación a las crisis en términos sociales, económicos y políticos que han acontecido en nuestro país. Ahora bien, más allá de todo esto, los resultados aquí mostrados ponen en evidencia una reflexión bastante clara: el estado que vive la producción científica en torno a la preeclampsia en nuestro país. Siendo la preeclampsia la segunda causa de mortalidad materna directa en el país, el estado de la investigación en este terreno resulta siendo, más que paradójico, escaso.

Por otro lado, para profundizar más en el estado de la cuestión, resulta importante resaltar que el $34,3 \%$ de las publicaciones analizadas se realizaron a partir de una sola investigación, de tipo caso-control y llevada a cabo entre los años 1997 y 1998 por S. Sánchez y colaboradores. El hecho que 11 publicaciones se basen en una sola base de datos demuestra no solo lo poco que se investiga en el Perú, sino también: por un lado, el alto nivel de especificidad de las publicaciones, y lo que es peor: que las publicaciones en este campo abordan aspectos específicos en lugar pretender abordar aspectos generales o panorámicos. Siendo tan escaso el conocimiento de la realidad de la preeclampsia en el Perú, investigar cosas puntuales podría significar investigar de espaldas a la realidad del país. Esto, tratándose la preeclampsia de la segunda causa de muerte materna en el Perú, no deja de llamar la atención. Por otro lado, podría decirse también que este nivel de explotación de una sola base de datos podría evidenciar el afán de la investigación en este campo de estudio (hecho que tendría que verificarse a la luz del estudio en otros campos): un afán academicista, dirigido al incremento del prestigio en el investigador, mas no un afán comprometido, dirigido a lograr un cambio real y concreto en el fenómeno que se estudia (para lo cual se necesitan no solo datos sino teorías, no visiones específicas sino visiones sistemáticas). 
Se exploró, además, el tiempo transcurrido entre el envío del artículo a la revista hasta su publicación, en el $50 \%$ de artículos, encontrándose que el tiempo promedio fue 4,5 meses. Sin embargo, aunque de utilidad, este dato no fue especificado en las revistas peruanas. Por otro lado, en lo que respecta al tiempo de demora de publicación desde la finalización del estudio hasta su respectiva publicación, se encontró que el promedio fue de 3,6 años; en comparación con los 2,8 años en promedio en el estudio bibliométrico de $\mathrm{VIH} / \mathrm{SIDA}$ y 2,9 años en el de tuberculosis. Considerando que el máximo tiempo de demora fue de 8 años, son datos pueden dar luces acerca de la prioridad que le dan los investigadores al envío de sus respectivas publicaciones referentes al tema de preeclampsia.

Se comprueba que existe aún una mirada centralista en esta área de la investigación, hallándose un $81,25 \%$ de estudios realizados netamente en la capital. La importancia de señalar este dato recae en que esta problemática afecta en mayor medida a las mujeres que residen al interior del país ${ }^{(18)}$. Es preciso promover la actividad científica a nivel nacional, no sólo por constituir una de las prioridades nacionales de investigación, sino también porque implementando políticas claras se protegerían más eficazmente a las poblaciones vulnerables.

Analizar el factor de impacto de las revistas en las que se encuentran estas investigaciones brinda una medida de la repercusión que éstas han tenido en la comunidad científica en los años posteriores a su publicación. De las revistas en que se pudo calcular dicho valor, se encontró que no hubo publicación con un factor de impacto mayor a 4.780 , siendo 2.696 el promedio de todas éstas. Esto difiere claramente a lo obtenido en un estudio bibliométrico nacional sobre tuberculosis ${ }^{(15)}$, en el que por lo menos 3 revistas en las que se publicó tenían un factor de impacto de más de 10. Estos datos pueden prestarse a discusión en cuanto a la calidad de las publicaciones nacionales sobre preeclampsia, las que pueden no cumplir con los estándares académicos que exigen las revistas con mayor índice de impacto. Otra razón, es que no existe interés por parte de estas revistas en publicar sobre preeclampsia. Respecto a este último dato, se procedió a realizar una búsqueda simple de los términos "Preeclampsia/ eclampsia" y "Tuberculosis" en las revistas biomédicas con mayor repercusión científica "New England Journal of Medicine" y "The Lancet", encontrándose una resaltante diferencia en la cantidad de resultados. Para las primeras palabras de búsqueda se encontraron 49 y 8 resultados, mientras que para el tema tuberculosis, se encontró 1313 y 3657 en ambas revistas respectivamente. En todo caso, el interés por parte de las revistas podría despertarse a la luz de una investigación creativa y que de luces vivas a la solución de un problema, cosa que tal como se ha mostrado no sucede con las investigaciones sobre preeclampsia en el Perú.

A la luz de estos resultados, cabe llamar a reflexión acerca de las causas de la reducida producción científica en nuestro medio que, según la UNESCO ${ }^{(19)}$, una posible explicación podría ser la falta de presupuesto que se pone a disposición en este campo biomédico pero sin duda también el escaso interés que muestran los profesionales médicos en hacer de la investigación un instrumento eje de su práctica diaria.

Entre las limitaciones encontradas al realizar el estudio, se encuentra el sesgo de publicación por el cual no todas las investigaciones logran publicarse o estar disponibles, para que puedan servir de base para la elaboración de otros estudios. Esto debido a la rigurosidad de las bases de datos internacionales en las cuales las revistas no son indexadas por no cumplir ciertos criterios de inclusión.

Finalmente, se pretende con el presente estudio bibliométrico, visibilizar la urgencia por impulsar la investigación sobre preeclampsia y otros diagnósticos en salud materna que puedan ser base de la generación de políticas que posibiliten una mayor protección en salud de la población peruana.

Financiamiento: Recursos propios.

Conflictos de Interés: Autores no declaran ningún conflicto de interés.

Autor corresponsal: Oscar Huapaya Huertas

Av. Roosevelt 877 Int. 2 Santiago de Surco.

981063805. Correo electrónico: oscarh12@gmail.com

\section{REFERENCIAS BIBLIOGRÁFICAS}

1. Hawfield A, Freedman BI. Pre-eclampsia: the pivotal role of the placenta in its pathophysiology and markers for early detection. Ther Adv Cardiovasc Dis. 2009;3(1):65-73.

2. Cavanagh $D$, Knuppel R. Obstetricia y perinatología. principios y práctica. Primera ed. Iffy L, Kaminetzky HA, editors. Buenos Aires: Editorial Médica Panamericana; 1992. 1276-80 p.

3. National High Blood Pressure Education Program Working Group on High Blood Pressure in P. Report of the National High Blood Pressure Education Program Working Group on High Blood Pressure in Pregnancy. American Journal of Obstetrics and Gynecology. 2000;183(1):S1-S22.

4. American-College-of-Obstetricians-and-Gynecologists. ACOG practice bulletin. Diagnosis and management of preeclampsia and eclampsia. Int J Gynaecol Obstet. 2002;77(1):67-75.

5. Pinheiro MdB, Junqueira DRG, Coelho FF, Freitas LG, Carvalho MG, Gomes KB, et al. D-dimer in preeclampsia: Systematic review and meta-analysis. Clinica Chimica Acta. 2012;414(0):166-70.

6. Pritchard J, MacDonald P, Gant N. Williams Obstetricia Tercera edición Barcelona: Salvat Editores. 1990.

7. Redman CWG, Roberts JM. Management of preeclampsia. Lancet. 1993;341:1451-4.

8. Naljayan MV, Karumanchi SA. New Developments in the Pathogenesis of Preeclampsia. Advances in Chronic Kidney Disease. 2013;20(3):265-70.

9. Sanchez S QC, Williams M, Lam N, Sorensen T. Headaches and Migraines Are Associated With an Increased Risk of 
Preeclampsia in Peruvian Women. American Journal of Hypertension. 2008;21(3):360 - 4.

10. Ministerio-de-Salud. Análisis y Situación de la Salud. Boletin Epidemiológico 2012;21(26)

11. Ministerio-de-Salud. Documento Técnico: Plan Estratégico Nacional para la Reducción de la Mortalidad Materna y Perinatal 2009 - 2015. 2009.

12. Caballero $P$ YM, Espinoza $M$, Castilla $T$, Granados $A$, Velazquez A, et al. . Prioridades regionales y nacionales de investigación en salud, Perú 2010-2014: Un proceso con enfoque participativo y descentralista. Rev Peru Med Exp Salud Publica. 2010;27(3):398-411.

13. Instituto-Nacional-de-Estadistica-e-Informatica. Encuesta Nacional de Hogares 2012. 2012.

14. Rehn C, Kronman U. Bibliometric handbook for Karolinska Institutet. Huddinge: Karolinska Institutet. 2008.

15. Yagui-Moscoso M, Oswaldo-Jave $H$, Curisinche-Rojas M. Análisis bibliométrico de la investigación sobre tuberculosis en el Perú: periodo 1981-2010. An Fac med. 2012;73(4):299-306.

16. Caballero P GC, Rosell G, Yagui M, Alarcón J, Espinoza M, Magan C, Sebastián C, Cabezas C, Romaní F. Análisis Bibliométrico de la producción Científica sobre VIH/SIDA en el Perú 1985 - 2010. Rev Peru Med Exp Salud Publica. 2011;28(3):470-6.

17. Taype-Rondán Á, Lajo-Aurazo Y, Huamaní C. Producción científica peruana sobre trastornos mentales en SciELOPerú, 2006-2011. Rev Med Hered. 2012;23(3):166-71.

18. Amarán Valverde JE, Sosa Zamora M, Pérez Pérez M, Arias Acosta D, Valverde Bravo I. Principales características de la preeclampsia grave en gestantes ingresadas en un hospita de Zimbabwe. Medisan. 2009;13(3):0-

19. Michán L, Llorente-Bousquets J. Bibliometría de la sistemática biológica sobre América Latina durante el siglo XX en tres bases de datos mundiales. Revista de Biología Tropical. 2010;58(2):531-45.

ORCID iDs

Oscar Antonio Limay Ríos

https://orcid.org/0000-0001-6012-3705 